\title{
Method for the Design of Broad Energy Range Focusing Reflectrons
}

\author{
Paul R. Vlasak, Douglas J. Beussman, Qinchung Ji, and Christie G. Enke \\ Department of Chemistry, Michigan State University, East Lansing, Michigan, USA
}

\begin{abstract}
A novel method for the design of reflectrons capable of focusing large kinetic energy ranges is presented. The design method itself is a numerical approach that provides a geometrically flexible alternative to traditional analytical design solutions. This design method has been used to produce a reflectron that provides unit mass resolution for product spectra in a tandem reflectron time-of-flight (TOF) mass spectrometer despite a kinetic energy range of $1950-2700 \mathrm{eV}$. In this application, the systematic progression of reflectron design results in a practical, nonlinear field reflectron with the use of only two grids. Design improvements are proposed for more flexible systems, although geometric constraints in the current instrument limit their experimental evaluation. (J Am Soc Mass Spectrom 1996, 7, 1002-1008)
\end{abstract}

$\mathrm{I}$ n reflectron time-of-flight (TOF) mass spectrometers that employ electron ionization sources, the application of an extraction field to the source volume creates a minimal spatial-temporal distribution for isomass ion packets outside of the ion source at a position called the space-focus plane [1-4]. These ion packets have a kinetic energy distribution as a result of their initial spatial and energy distributions. The reflectron, or ion mirror, is used to reproduce the spatial-temporal distribution of the space-focus plane at a second position, such as the surface of an ion detector, which thereby improves mass resolution by increasing flight time (and the separation between adjacent isomass ion packets) without increasing significantly the temporal width of the isomass ion packets [2-6]. Generally, the relative kinetic energy range of ions accelerated from the source is small $(<10 \%)$, and most reflectron time-of-flight instruments described in the literature have been designed to focus these small energy ranges effectively.

The most commonly used reflectrons are one- and two-stage designs that provide first- and second-order energy compensation, respectively. Homogeneous electric fields (stages) are created with the use of wire mesh "grids." The prescribed geometry of the reflectron field, in both cases, is an analytically derived function of instrument variables $[2,5]$. Several grid-free reflectron designs also have been described [6-11]. The ability to focus energy without the use of grids is considered an advantage because grids limit transmission. Also, the cylindrically symmetric nonhomogeneous reflectron field can be configured, through considerable effort, to focus the ion beam radially. Grix et al. [6] report that an energy range of greater than $10 \%$

Address reprint requests to Dr. C. G. Enke, Department of Chemistry, 103 Clark Hall, University of New Mexico, Albuquerque, NM 87131. can be focused effectively by using a grid-free design. Rockwood [12] developed a method that uses a parabolic field in a device termed a perfectron to give infinite-order correction. The perfectron has no field free region and can, theoretically, temporally focus ions of all energies that are reflected by its field. Unfortunately, implementation of this solution is difficult because the curved nature of the axial perfectron field results in a curved radial field, which causes beam divergence. When the field-free distance is set to zero, the calculation used in the design method described here yields a parabolic curve essentially identical to the perfectron solution. More recently, Cornish and Cotter $[13,14]$ described a curved field reflectron arrived at by application of a slight curvature to the conventional one-stage design. They described ways to effectively minimize the divergent effects of the curved radial field and showed that $90 \%$ of the energy range reflected by this device can be focused simultaneously. When the instrument parameters for the calculation that follows are chosen to approximate the geometry of Cornish and Cotter's instrument, this calculation gives a similar solution.

The platform from which we will demonstrate this design method is the tandem reflectron time-of-flight (tandem TOF) mass spectrometer constructed in our laboratory $[15,16]$. In this instrument, precursor ion packets (that have a small relative energy spread) are focused by a first reflectron to an interaction region, where the ions are fragmented selectively by pulsedlaser photon-induced dissociation (PID). The resulting ions have a large kinetic energy range because the fraction of precursor ion energy retained by a product ion is equal to the fraction of the mass retained by the product, where any kinetic energy release due to the fragmentation process is neglected. The average acceleration energy from the ion source is $650 \mathrm{eV}$, and the 
resulting product ions can have energies between nearly 0 (for very low mass-to-charge ratio product ions) and $650 \mathrm{eV}$ (for unfragmented precursor ions). After dissociation, product ions are accelerated by an additional $1950 \mathrm{~V}$ to induce mass-to-charge ratio-dependent velocities and to reduce the relative kinetic energy spread. The spatial-temporal distribution of product ions produced in the interaction region by PID of an isomass precursor ion packet is the distribution to be reproduced at a detector by the second novel reflectron [17]. Although the example used in this discussion is the focusing of product ions accelerated after dissociation, this method can be applied to reflectron design for normal TOF mass spectrometry as well.

\section{Reflectron Design Process}

The reflectrons with which we will illustrate our design process contain a linear field region and a nonlinear field region. A calculation is used to obtain a theoretical voltage profile for the nonlinear field region based on instrument parameters. This profile is generated as a series of linear segments, such that the nodes of the segmented curve correspond to the positions of virtual grids in a theoretical reflectron. The potential applied to each grid is calculated so that isomass ions that have exactly enough energy to reach a grid, stop there, and when they change direction they will have the same total flight time as isomass ions that reach each of the other grids. Ions that turn around at positions other than the grid locations will have slightly different total flight times.

Suppose the lowest energy ion that we are interested in focusing has energy $U_{1}$. Our strategy is to construct the first reflectron segment from two parallel grids placed a distance $L_{1}$ apart so that an ion that has energy $U_{1}$ will stop exactly at the second grid. To achieve this, the field-free voltage is applied to the first grid and a voltage $P_{1}$ is applied to the second grid:

$$
P_{1}=\frac{U_{1}}{z} \cos ^{2} \alpha
$$

where $\alpha$ is the angle between the flight path in the field-free region and the axis of the reflectron, and $z$ is a single positive charge.

The length of the flight path outside of the reflectron (field-free region) is $L_{\mathrm{ff}}$, which is the sum of the distance from the initial space-focus plane to the face of the reflectron and the face of the reflectron to the reflectron focal point (usually the detector position). The flight time $(t)$ of a hypothetical singly charged positive ion that has energy $U_{1}$ can be calculated from

$$
t=\frac{L_{\mathrm{ff}}}{v_{\mathrm{ff}}}+\frac{2 L_{1}}{\bar{v}_{1}}
$$

where $v_{f f}$ is the velocity of the ion in the field-free region given by

$$
v_{\mathrm{ff}}=\sqrt{\frac{2 U_{1}}{m}}
$$

where $m$ is the mass of the ion. The average velocity $\left(\bar{v}_{1}\right)$ of the ion in the space between grids one and two (parallel to the reflectron axis) is given by

$$
\bar{v}_{1}=\frac{1}{2} v_{\mathrm{ff}} \cos \alpha
$$

All terms in eq 2 (fully expanded) are known or measurable physical quantities with the exception of $m$, which may be chosen arbitrarily as long as its value is kept constant for all further calculation steps.

Once we establish a flight time $(t)$ for an ion of energy $U_{1}$, we wish to establish a second field between grid two and a third grid placed a distance $L_{2}$ behind grid two. The flight time for an ion that stops at this third grid is given by

$$
t=\frac{L_{\mathrm{ff}}}{v_{\mathrm{ff}}}+\frac{2 L_{1}}{\bar{v}_{1}}+\frac{2 L_{2}}{\bar{v}_{2}}
$$

where the velocity terms are now

$$
\begin{aligned}
& v_{\mathrm{ff}}=\sqrt{\frac{2\left(U_{1}+U_{2}\right)}{m}} \\
& \bar{v}_{1}=\frac{1}{2}\left[v_{\mathrm{ff}} \cos \alpha+\sqrt{\frac{2 U_{2}}{m}} \cos \alpha\right] \\
& \bar{v}_{2}=\frac{1}{2} \sqrt{\frac{2 U_{2}}{m}} \cos \alpha
\end{aligned}
$$

where $U_{2}$ is the additional energy that the ion must have (above $U_{1}$ ) to exactly reach grid three. By using the flight time $(t)$ calculated from eq 2, all terms in Equation 5 (fully expanded) are known values with the exception of $U_{2}$ which we solve for numerically using a program called MathCad ${ }^{\circledR}$ [18].

The remainder of the reflectron field segments are calculated by using MathCad according to the general equation for the $n$th stage $(n>2)$ :

$$
t=\frac{L_{\mathrm{ff}}}{v_{\mathrm{ff}}}+\sum_{i=1}^{n-1} \frac{2 L_{i}}{\bar{v}_{i}}+\frac{2 L_{n}}{\bar{v}_{n}}
$$

where the general velocity terms are

$$
\begin{aligned}
v_{\mathrm{ff}} & =\sqrt{\frac{2 \sum_{j=1}^{n} U_{j}}{m}} \\
\bar{v}_{i} & =\frac{1}{2}\left[v_{\mathrm{ff}} \cos \alpha+\sqrt{\frac{2 \sum_{j=i+1}^{n} U_{j}}{m}} \cos \alpha\right]
\end{aligned}
$$




$$
\bar{v}_{n}=\frac{1}{2} \sqrt{\frac{2 U_{n}}{m}} \cos \alpha
$$

$P_{n}$ (for all values of $n$ ) is found according to

$$
P_{n}=\frac{U_{n}}{z} \cos ^{2} \alpha
$$

For our tandem TOF instrument, we have calculated a potential profile for the second reflectron that contains 12 segments ( 13 grids) by using the foregoing method. This reflectron was configured for a drift length $\left(L_{\mathrm{ff}}\right)$ of $188.6 \mathrm{~cm}$, the distances between the grids $\left(L_{1}\right.$ to $L_{12}$ ) as given in Table 1 , the angle $(\alpha)$ between the flight path and the reflectron axis of $2^{\circ}$, $1650 \mathrm{eV}$ as the lowest energy that need be focused $\left(U_{1}\right)$, and $2700 \mathrm{eV}$ as the highest energy that can be focused. The actual calculated potentials to be applied to the grids (relative to the field-free potential) and the energies of hypothetical ions that reach each grid are given in Table 1. Generally, the profile is curved through the first several grids and becomes more linear toward the back of the reflectron. To obtain a more exact field curvature, electrodes were placed more densely in the initial curved portion of the reflectron. The shape of the calculated profile depends on the values chosen for $L_{\mathrm{ff}}, U_{1}$, and $L_{1}-L_{12}$. The profile used here is the result of several iterations of input parameters, restricted by the physical dimensions of our vacuum chamber.

The flight times for ions that have energies that cause them to turn around between electrode positions can be calculated. Figure 1 is a plot of $2 \Delta t / t$ (where $\Delta t$ is the difference between the flight time for a particular ion and the time for an ion that turns around at a grid position) versus ion energy for the calculated reflectron field profile. Calculations were made at $1-\mathrm{eV}$ intervals for plot preparation purposes. Positions where the curve dips toward zero indicate grid locations. In the front of the reflectron (where the curvature of the voltage versus distance profile is greatest) $2 \Delta t / t$ ex-

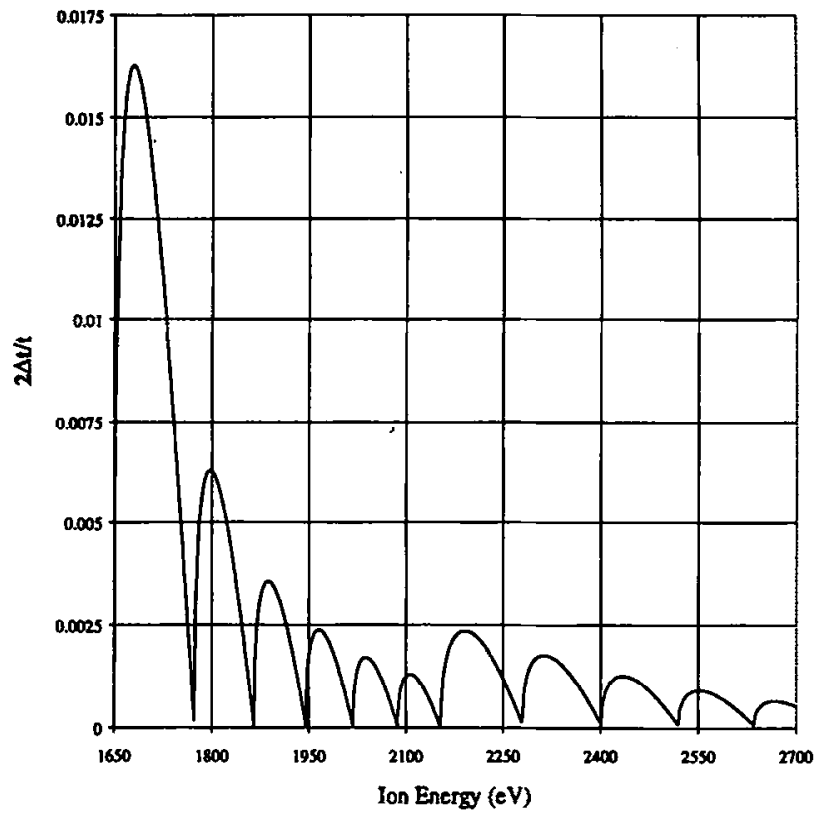

Figure 1. Inverse resolution $[t /(2 \Delta t), F W H M]$ versus ion energy for the calculated reflectron field profile determined by MathCad.

ceeds $0.016(R \sim 50)$. In the back of the reflectron, where the voltage versus distance profile is more linear, the flight time deviations for ions that turn around between grids are smaller. However, there is a jump to larger deviations between 2100 and $2200 \mathrm{eV}$. This jump corresponds to the change from closely spaced grids in the front of the reflectron to more widely spaced grids in the back (see Table 1).

To develop an effective focusing profile, we set $U_{1}$ to a value $300 \mathrm{eV}$ lower than the lowest possible energy for the product ions to take advantage of the smaller deviations in the back of the reflectron. In Figure 1, the portion of the reflectron in which ions turn around is from 1950 to $2700 \mathrm{eV}$. Notice that $2 \Delta t / t$ is less than $0.0025(R>400)$ for this energy

Table 1. Reflectron segment configuration

\begin{tabular}{lcccc}
\hline Segment & $\begin{array}{c}\text { Length }^{8} \\
(\mathrm{~cm})\end{array}$ & $\begin{array}{c}\text { Energy of ion } \\
(\mathrm{eV})\end{array}$ & $\begin{array}{c}\text { Calculated } \\
\text { potential }^{\circ}(\mathrm{V})\end{array}$ & $\begin{array}{c}\text { Adjusted } \\
\text { potential }^{\mathrm{d}}(\mathrm{V})\end{array}$ \\
\hline \hline 1 & 1.054 & 1650.00 & 1647.99 & 1647.99 \\
2 & 0.527 & 1773.79 & 1771.63 & 2590 \\
3 & 0.527 & 1865.44 & 1863.17 & 1910 \\
4 & 0.527 & 1945.10 & 1942.73 & 1920 \\
5 & 0.527 & 2018.35 & 2015.89 & 2015 \\
6 & 0.527 & 2087.51 & 2084.97 & 2095 \\
7 & 0.527 & 2153.81 & 2151.19 & 2175 \\
8 & 1.054 & 2279.87 & 2277.09 & 2278 \\
9 & 1.054 & 2401.28 & 2398.36 & 2403 \\
10 & 1.054 & 2519.53 & 2516.46 & 2526 \\
11 & 1.054 & 2635.59 & 2632.38 & 2635.5 \\
12 & 1.054 & 2750.14 & 2746.79 & 2745 \\
\hline
\end{tabular}

\footnotetext{
- Distances of linear field regions between grids.

benergies of ions that would exactly reach each grid.

c Calculated potentials for the fully gridded reflectron.

${ }^{d}$ Empirically adjusted potentials used in the final two-grid reflectron simulation.
} 
range. This resolution is limited by the spacing between the grids. Extremely high resolution (a nearly perfect solution) could be achieved if infinitesimally small segments were used for the reflectron construction. In fact, since this work was completed, an analytical solution that can define the curve exactly has been described [19]. In our case, the distances between electrodes (and hence the number of electrodes) were determined by the dimensions of available construction materials. The predicted resolution for a two-stage reflectron over this energy range is about 250 [5].

We next constructed a model of the 12-stage reflectron in SIMION-a program designed to predict electric fields based on position, shape, and voltage applied to graphically entered electrodes and to predict the motion of charged particles in the fields produced [20]. Figure 2a is the SIMION reflectron; the heavy lines indicate electrodes and the light lines indicate equipotential contours. The reflectron is cylindrically symmetric. Grid one is on the left, with the solid back plate at the right. A shroud extends from the first grid around the other higher potential electrodes to preserve the field-free drift space. The potentials that correspond to the equipotential contour lines are the calculated potentials as given in Table 1 . The contours follow the conductive electrodes, with minimal leakage of the field-free potential into the spaces between the electrodes near the edges.

Figure 3a shows the SIMION trajectories of 6 isomass ions that have kinetic energies of 1950, 2100, $2250,2400,2550$, and $2700 \mathrm{eV}$. These ions begin at the same point (a perfect space-focus plane at upper left) with a trajectory $2^{\circ}$ from the reflectron axis. The low energy ions do not penetrate the reflectron field as far as the high energy ions, have a smaller velocity component perpendicular to the reflectron field, and spend less time in the reflectron. When the isomass ions exit the reflectron, they follow parallel paths and eventually become focused temporally at a second space-focus plane. The tick marks (triangles) in the ion trajectories are equal time markers. Figure $3 a^{\prime}$ is an expanded view of the portion of Figure 3a contained in the rectangle.

In the SIMION simulation, the grids are assumed to have $100 \%$ ion transmission. However, because the grids actually have a limited open area (88\%), the transmission of ions through 12 grids that both enter and exit the reflectron is only about $5 \%$. Also, field distortions in the vicinity of grids can result in deflection of ions, which further contributes to transmission loss and peak broadening [21]. To avoid this limitation, we replaced the virtual grids in the SIMION reflectron with open rings with the exception of the first two grids (which define the end of the field-free region and the steep deceleration region) and the solid plate at the back of the reflectron. Figure $2 b$ is a SIMION plot of the equipotential contours that result from this new configuration by using the calculated potentials for the contours as given in Table 1 . The contours deviate

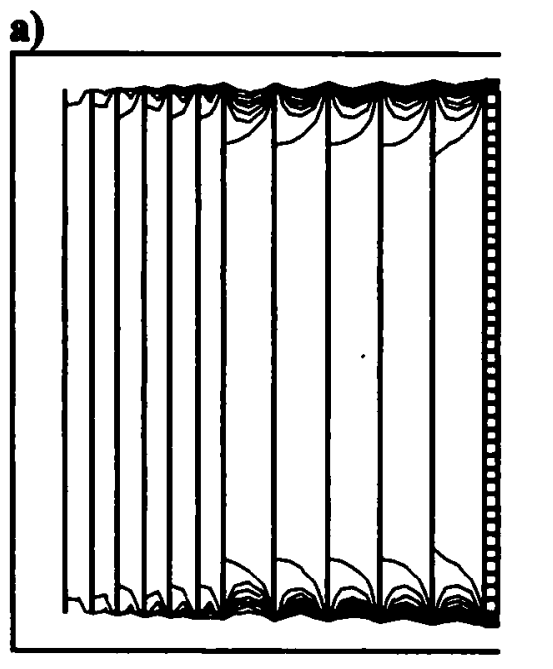

b)

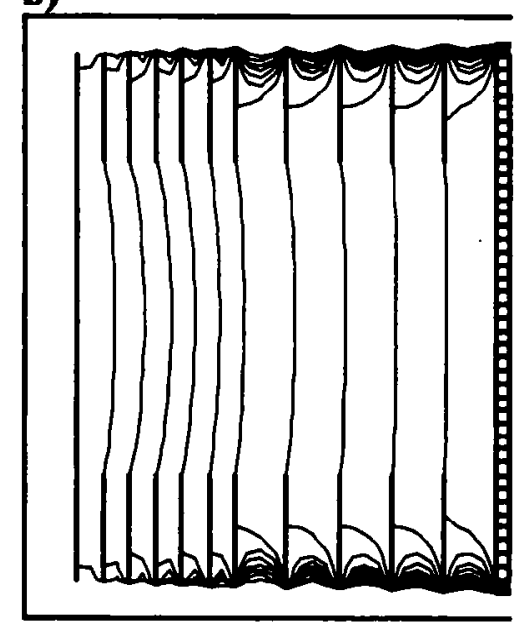

c)

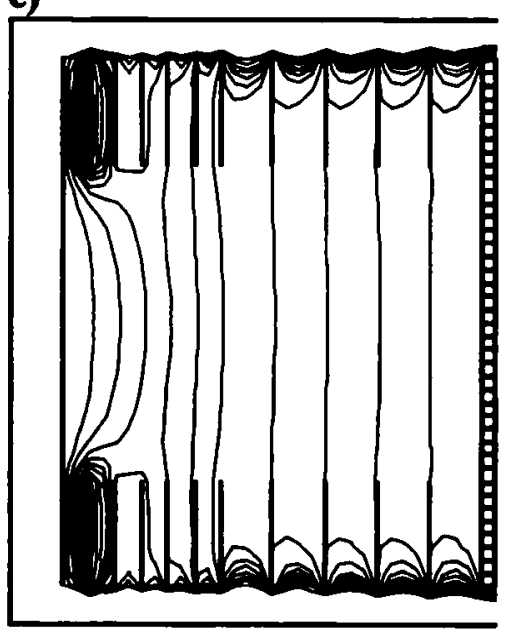

Figure 2. SIMION plots that show reflectron electrodes (heavy lines) and equipotential contours (light lines) for (a) the totally gridded reflectron, (b) the reflectron from which all but the first two grids were replaced with ring electrodes, and (c) the two-grid reflectron after adjustment of the ring electrode potentials. The values of the equipotential contours are the calculated potentials given in Table 1. 


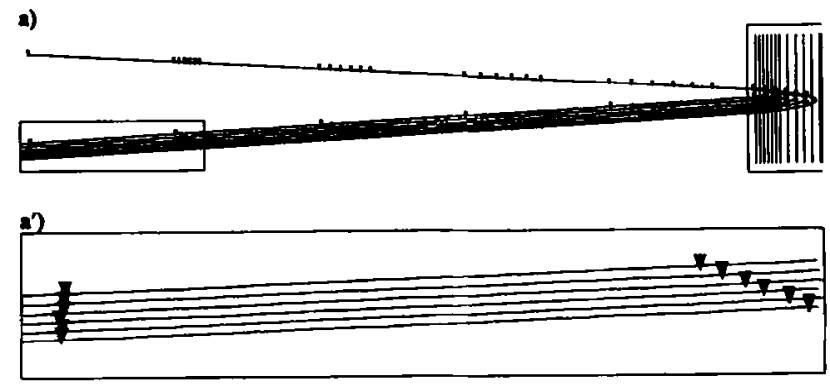

b)
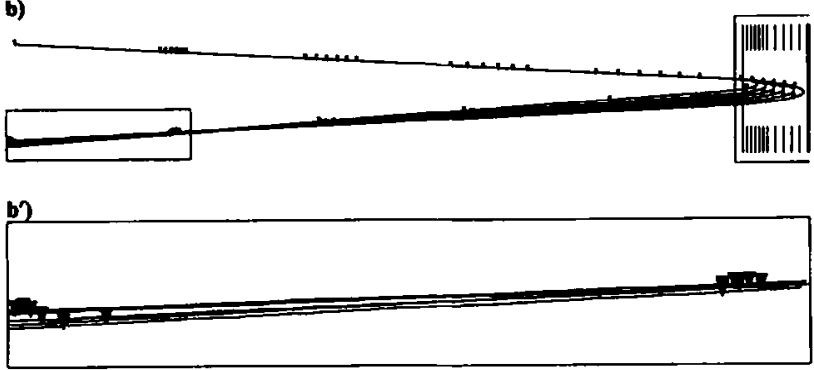

c)
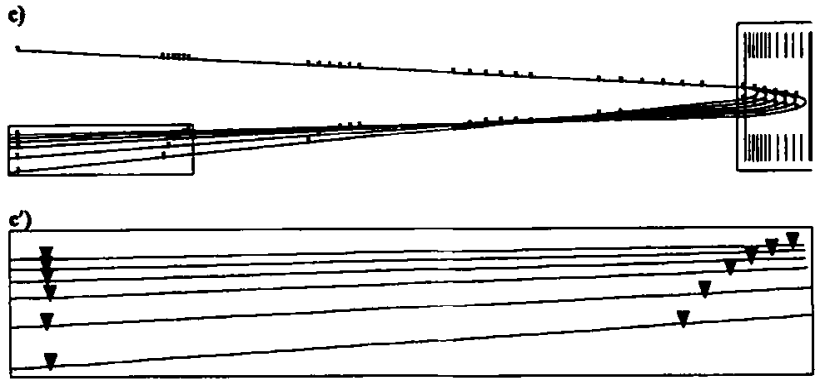

Figure 3. SIMION ion trajectory simulations for $\left(a, a^{\prime}\right)$ the totally gridded reflectron, $\left(b^{\prime}, b^{\prime}\right)$ the reflectron from which all but the first two grids were replaced with ring electrodes, and $\left(c, c^{\prime}\right)$ the two-grid reflectron after adjustment of the ring electrode potentials.

from the original grid position as the distance from the ring electrodes increases.

These field distortions result in three major consequences. First, the temporal focusing properties observed for the totally gridded reflectron simulation are degraded because the potential profile has changed. Second, the curved field affects radial ion motion, so that ions that enter the reflectron at different positions or at slightly different angles are steered differently (spherical aberration). Third, ions that enter the reflectron at the same position and angle, but have different energies, are steered differently (chromatic aberration). The loss of temporal focus that results from the field distortions can be observed in Figure $3 b$ and $3 b^{\prime}$. This reflectron yields a predicted mass resolution of less than 50 at the detector position.

To compensate for the field distortions seen in Figure $2 b$, the ring electrode voltages can be adjusted manually by an iterative empirical process. With each adjustment of electrode voltage, a contour plot is observed and trajectories are simulated to determine the effect. This procedure allows the calculated potential versus distance profile to be achieved quite closely in the two-grid reflectron for the path that the ions are expected to travel in the reflectron. Temporal focusing improves, but spherical and chromatic aberrations increase due to the increased curvature of the reflectron field. This can be seen in Figure 2c, which shows the equipotential contours for the manually adjusted reflectron. Use of only the center portion of the reflectron by reduction of $\alpha$ will reduce aberration effects. The adjusted voltages applied to the rings in this simulation are given in Table 1. Much of the compensation to the potential versus distance profile is accomplished by a large adjustment to the second segment (third electrode). Figure $3 c$ and $3 c^{\prime}$ show the improved temporal focusing as a result of the empirical adjustment. The effects of chromatic aberration in this case result in a broadened ion beam, which may result in ion loss due to the limited size of the detector face. In this application, if ion loss occurs, mass-to-charge ratio discrimination will occur because the aberration is a function of ion energy and ion energy is a function of mass-to-charge ratio of the product ions. Figure 4 is a plot of $2 \Delta t / t$ versus ion energy over the range of $1950-2700 \mathrm{eV}$ at intervals of $25 \mathrm{eV}$, which gives a theoretical resolution of approximately 800 . This configuration is used for the second reflectron in the tandem TOF instrument.

\section{Implementation, Results, and Discussion}

A photodissociation product spectrum obtained by using the two-grid empirically adjusted reflectron in the tandem TOF mass spectrometer is shown in Figure 5. The precursor ion was $m / z 91$ from toluene.

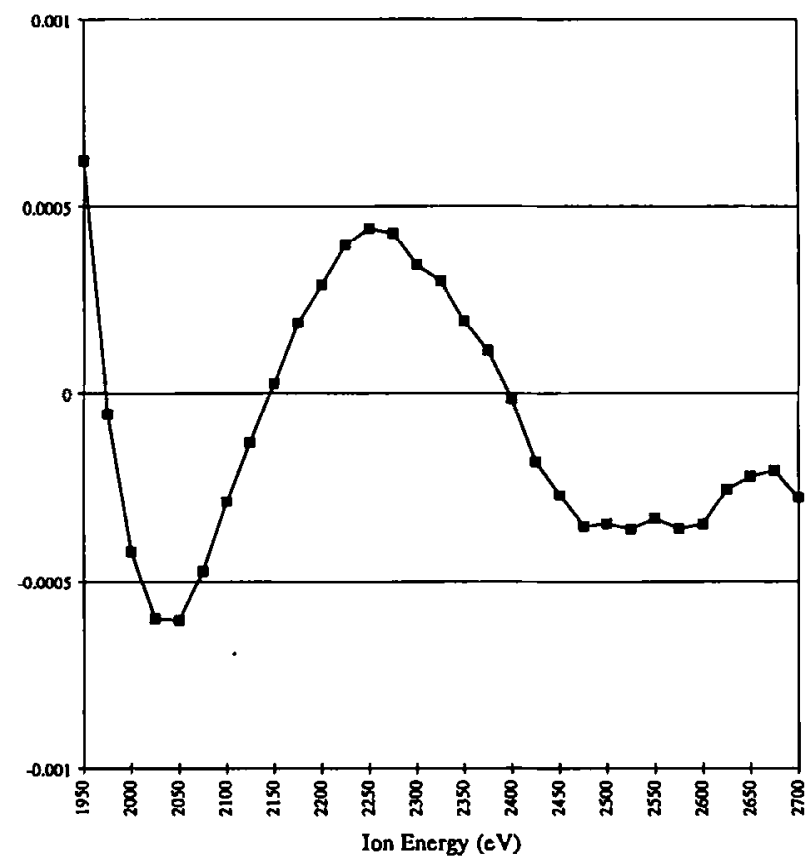

Figure 4. Inverse resolution [ $t /(2 \Delta t)$, FWHM] versus ion energy for the empirically adjusted reflectron as determined by SIMION. 


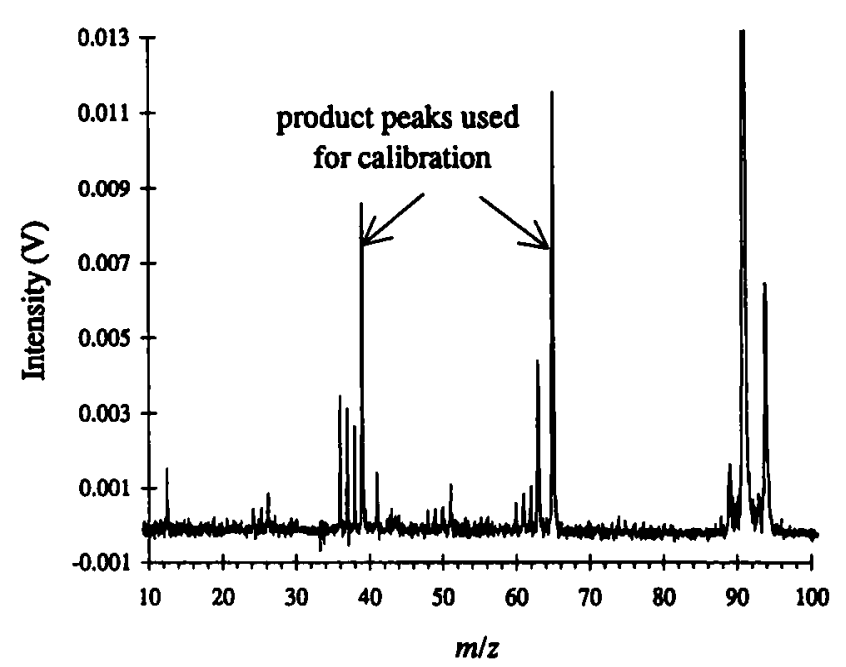

Figure 5. Product spectrum of $m / z 91$ of toluene collected by using the empirically adjusted two-grid reflectron (resolution of 300, FWHM).

Calibration of product spectra easily is accomplished by using the derived relationship

$t_{\text {prec }}-t_{\text {prod }}=\sqrt{\frac{m_{\text {prec }}}{A+B}}-\sqrt{\frac{m_{\text {prod }}}{\left[\left(m_{\text {prod }} / m_{\text {prec }}\right) A\right]+B}}$

where $t_{\text {prec }}$ and $t_{\text {prod }}$ are the precursor and product ion arrival times, $m_{\text {prec }}$ and $m_{\text {prod }}$ are the precursor and product mass-to-charge ratio values, and

$$
\begin{gathered}
A=\frac{2 \bar{U}_{\text {prec }}}{\left(L_{\text {ff }}\right)^{2}} \\
B=\frac{2 U_{\text {acc }}}{\left(L_{\mathrm{ff}}\right)^{2}}
\end{gathered}
$$

where $\bar{U}_{\text {prec }}$ is the average kinetic energy of the precursor ions and $U_{\text {acc }}$ is the energy gained by postdissociation acceleration. Because $A$ and $B$ are difficult to determine by direct measurement, two product ion arrival times for known mass-to-charge ratio values along with the arrival time and mass-to-charge ratio value of the precursor ion are used in eq 14 to solve for the two unknowns ( $A$ and $B$ ) by using MathCad. These constants can be used to calibrate product spectra collected from any mass-to-charge ratio value precursor ion for the same instrument tune. For the spectrum in Figure 5, the peaks at $m / z 39$ and 65 were used for calibration.

The mass resolution [ $m / \Delta m$ full width at half maximum (FWHM)] is about 300 for product spectra and about $1500[t /(2 \Delta t), F W H M]$ for normal spectra. This resolution for normal spectra through reflectron 2 (without laser photodissociation) is approximately the same resolution that is observed when a detector is placed at the focal point of the first reflectron. Ideally resolution would increase due to the increased flight time, but the effects of spherical aberration and the introduction of grids apparently limit resolution. Normal (undissociated) ion packets separate from one another continuously through the entire flight path beginning at the source, whereas product ion packets do not begin to separate from one another until they are accelerated after dissociation in the interaction region. For this reason the product spectra are not as well resolved as normal spectra. Because normal (undissociated) ion packets result in peaks that have approximately the same width as peaks due to product ion packets, we can conclude that broadening due to the kinetic energy of dissociation is not significant.

We also have considered a greater range for the independent variables in the design calculation than the dimensions of our vacuum chamber allow. We found that increasing the distance of the linear field region at the front of the reflectron resulted in a longer nonlinear region with less field curvature. Figure 6 is a comparison of two calculated profiles for the same choice of $U_{1}, \alpha$, and $L_{\mathrm{ff}}$ : curve $A$ is calculated by using $L_{1}=1.054 \mathrm{~cm}$ as was used previously in the tandem TOF reflectron 2 development; curve $B$ is calculated by using $L_{1}=10.54 \mathrm{~cm}$.

Because the profile for the reflectron with the longer first stage is less curved, the flight time deviations for ions that tum around between grid positions is smaller and there is less field distortion as a result of removal of the grids in the longer reflectron as compared to the shorter one, which reduces the extent to which ring electrodes need to be adjusted to restore the calculated profile. Also, the more homogeneous field of the longer reflectron causes less spherical and chromatic aberration. Finally, because the change in field strength across the reflectron grids is smaller for the longer linear stage than for the shorter linear stage, the detrimental

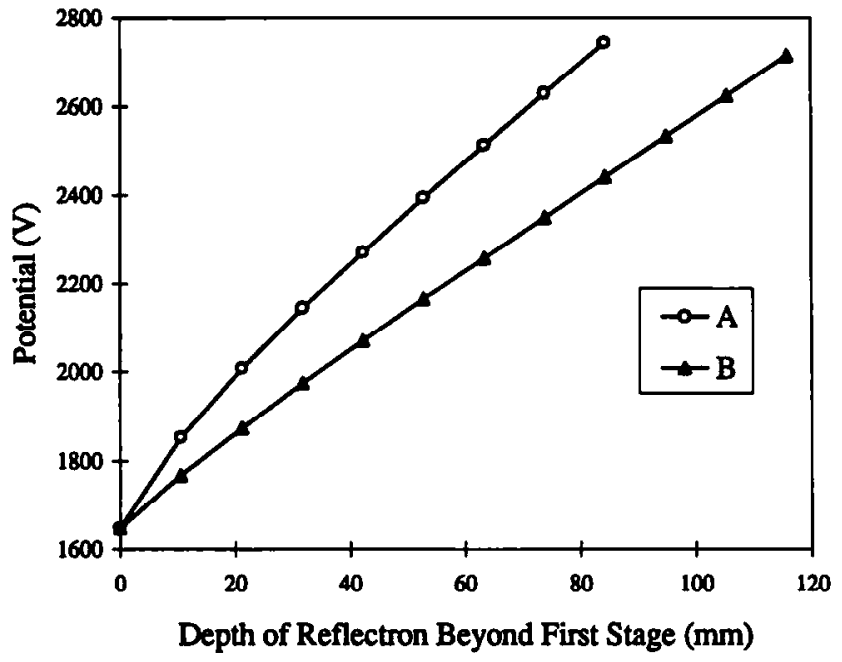

Figure 6. Curve $A$ is the calculated reflectron field profile (beyond the first stage) that used a first stage length $L_{1}=1.054 \mathrm{~cm}$. Curve $B$ is the calculated reflectron field profile (beyond the first stage) that used $L_{1}=10.54 \mathrm{~cm}$. 
field distortions in the vicinity of the reflectron grids will be smaller. Use of a smaller angle $\alpha$ is expected to result in improved performance. Although the nonlinear stage of the longer reflectron appears to be very nearly linear (curve B in Figure 6), approximation of this curve with a linear stage, in fact, results in a significantly less effective solution.

The particular reflectron design implemented in this instrument resembles, to some extent, the conventional two-stage design and the grid-free design described by Wollnik's group $[6,10]$. These reflectrons have a steep initial deceleration stage to negate the energy that all of the ions have in common. Following this deceleration there is a shallow field that serves to compensate for the time discrepancy that results from field-free flight of isomass ions of varying energies. These reflectrons are all relatively short compared to the field-free distance. The method described here also can be used to design longer reflectrons that do not employ a steep deceleration and resemble the curved field design described by Cornish and Cotter [13, 14]. In addition, in the limit of no field-free flight, the method described here gives a solution that very closely approximates the perfectron described by Rockwood [12]

\section{Acknowledgment}

We gratefully acknowledge the National Institutes of Health (NIH GM 44077 and NIH GM 49922) for partial support of this work.

\section{References}

1. Wiley, W. C.; McLaren, I. H. Rev. Sci. Instrum. 1955, 26, 1150-1157.

2. Karataev, V. I.; Mamyrin, B. A.; Shmikk, D. V. Sov. Phys. Tech. Phys. 1972, 16, 1177-1179.
3. Mamyrin, B. A.; Karataev, V. I.; Shmikk, D. V.; Zagulin, V. A. Sov. Phys.-JETP 1973, 37, 45-48.

4. Grix, R.; Gruner, U.; Li, G.; Stroh, H.; Wollnik, H. Int. J. Mass Spectrom. Ion Processes 1989, 93, 323-330.

5. Gohl, W.; Kutscher, R.; Laue, H. J.; Wollnik, H. Int. I. Mass Spectrom. Ion Processes 1983, 48, 411-414.

6. Grix, R.; Kutscher, R.; Li, G.; Gruner, U.; Wollnik, H. Rapid Commun. Mass Spectrom. 1988, 2, 83-85.

7. Berger, C. Int. J. Mass Spectrom. Ion Processes 1983, 46, 63-66.

8. Frey, R.; Weiss, G.; Kaminski, H.; Schlag, E. W. Z. Naturforsch., A: Phys., Phys. Chem., Kosmophys. 1985, 40, 1349-1350.

9. Walter, K.; Boesl, U.; Schlag, E. W. Int. J. Mass Spectrom. Ion Processes 1986, 71, 309-313.'

10. Kutscher, G.; Grix, R.; Li, G.; Wollnik, H. Int. J. Mass Spectrom. Ion Processes 1991, 103, 117-128.

11. Bergmann, T.; Martin, T. P.; Schaber, H. Rev. Sci. Instrum. 1990, 61, 2592-2600.

12. Rockwood, A. L. Proceedings of the 34th ASMS Conference on Mass Spectrometry and Allied Topics; Cincinnati, $\mathrm{OH}$, June 8-13, 1986; pp 173-174.

13. Cornish, T. J.; Cotter, R. J. Rapid Commun. Mass Spectrom. 1993, 7, 1037-1040.

14. Comish, T. J.; Cotter, R. J. Rapid Commun. Mass Spectrom. 1994, 8, 781-785.

15. Seeterlin, M. A.; Vlasak, P. R.; Beussman, D. J.; McLane, R. D.; Enke, C. G. J. Am. Soc. Mass Spectrom. 1993, 4, 751-754.

16. Beussman, D. J.; Vlasak, P. R.; McLane, R. D.; Seeterlin, M. A.; Enke, C. G. Anal. Chem. 1995, 67, 3952-3957.

17. Ji, Q.; Vlasak, P. R.; Beussman, D. J.; Enke, C. G. Proceedings of the 42nd ASMS Conference on Mass Spectrometry and Allied Topics; Chicago, IL, May 29-June 3, 1994; p 1039.

18. MathCad $5.0^{\circ}$ for Windows; (C) 1991-1994 by MathSoft, Inc., Cambridge, MA.

19. Flory, C. A.; Taber, R. C.; Yefchak, G. E. Int. J. Mass Spectrom. Ion Processes 1996, 152, 169-176; 177-184.

20. Dahl, D. A.; Delmore, J. E. SIMION PC/PS2 Version 5. 0 (real mode), Idaho Falls National Engineering Laboratory, EG \& G Idaho Inc., Idaho Falls, ID, 1988.

21. Bergmann, T.; Martin, T. P.; Schaber, H. Rev. Sci. Instrum. $1989,60,347-349$. 